\title{
Barcode Recognition Using a Morphological Analysis
}

\author{
Wen-Yen Wu
}

\begin{abstract}
In this paper, we propose a simple method to recognize barcodes. It is based on the feature extraction of lines from the barcodes. It firstly captures the gray-scaled images by CCD camera. Then the regions of bars and spaces will be separated, and the widths of barcodes are then recorded. Finally, we use the feature of widths to recognize the barcodes. From the experimental results, it can be found that the proposed method is effective for bar code recognition.
\end{abstract}

Index Terms - barcode, recognition, feature extraction, morphlogical analysis.

\section{INTRODUCTION}

Barcode has been used for many years and has been recognized by all walks of life. Early bar code changes were limited to the application of coding rules or the development of custom rules, so many types of bar codes have been born, such as 39 codes, EAN codes, 128 codes, etc. Until recent years, a new two-dimensional barcode technology has gradually developed. Two-dimensional barcodes can not only take advantage of the characteristics of one-dimensional barcodes, but also increase the amount of data carried by the original barcode, and will perform self-checking for errors and errors. Repair actions, such features are very helpful to the expansion of barcode application environment and system flexibility. However, because the rules are not unified, the application or practicality still needs to be considered, and it has not yet reached the popularization of one-dimensional barcodes.

On the other hand, today's barcode reading technology is still mostly limited to barcode readers. The biggest disadvantage is that when identifying barcodes, the object must be close to the barcode reader for a certain distance in order to perform the reading action. However, in recent years, some scholars have developed laser light, using the principle that red light reacts strongly to contrasting colors, so that barcodes do not have to be very close to the object to obtain identification results or barcode information as in the past. However, in practical applications, due to the high price of laser light, the increase in equipment cost and low versatility. Therefore, some scholars later applied magnetic induction in the field of barcodes to magnetize the material of the barcode, which can make the reading method simpler. Users can obtain barcode information without locking the barcode machine to a specific area, which improves the convenience

Wen-Yen Wu, Department of Industrial Management, I-Shou University, Kaohsiung, Taiwan of the barcode. This method is widely used in book management and hypermarket or shopping mall management. However, the above methods have a common disadvantage, that is, the barcode reader and the object barcode must be close to a certain distance to be able to be identified. If the barcode reader needs to be read at a longer distance, it will still be difficult to read.

In recent years, some scholars have studied to directly capture images by image capture devices, locate the position of the barcode, and then identify the information contained in the barcode. This method not only expands the application range of barcodes, but also improves the inconvenience caused by the need to approach the barcode when the barcode reader is reading. Aiming at finding the barcode in the image, it is proposed to detect the gradient changes of the four angles in the image to highlight The barcode image is output, and then the barcode area in the image is searched out by using the characteristics of the barcode with high density of black and white in the area, the single directionality of the barcode and the height of the barcode.

Nowadays, the applications of machine vision technology are very popular. It can reduce the unnecessary manpower expenses and provide a fast and convenient application. There are many kinds of barcodes, they include UPC/EAN, Code 39, Code 128, Interleaved 2 of 5, and etc. [1, 5, 6]. In generally, each type of barcodes has its own advantage.

Amould et al. [1] uses the black and white feature of the barcode, that is, from the perspective of $3 \mathrm{D}$, it is like mountains and valleys. This feature is used to determine the gray scale of the barcode to eliminate noise, and then to do the image. A series of morphological operations find the position of the barcode in the original image.

Jain \& Chen [3] used Gabor calculations to filter out the textures in the image from four angles, and then used two methods to extract barcodes from the image: the first is an unsupervised search method. Use the points on the screen after the texture is filtered out to do the clustering action, and save the barcode image by removing too many or too few groups. The other is the supervised bar code search method, which finds the bar code through the vertical sensing of the known position and the strongest response by computing. So far, it is not difficult to find that many papers are aimed at highlighting the bar code by the obvious color difference between the bar code and the surrounding environment.

Muniz et al. [5] used the Holstein transformation method to detect the position of the bar code, because the Holstein transformation can detect a straight line or repair the straight line to locate the bar code in a thick position, and then recognize it. The biggest disadvantage of conversion is that 
the amount of calculation is huge and the processing is too time-consuming.

Viard-Gaudin et al. [8] enhanced the four-direction gradient of the image, used the combination of morphological calculations to filter out irrelevant noise and objects, and then used the projection process to easily obtain the barcode in the image

In recent years, the application of neural networks has solved many problems that were not easy to solve in the past, so some scholars have applied neural networks in bar code extraction in images. Howlett et al. [2] hope to solve the problem of difficult control of the barcode image capturing environment by using the two characteristics of neural network to be more resistant to environmental noise and being able to recognize broken information. After experimenting with network structures such as MLP and $\mathrm{RBF}$, it was found that the specifications and sizes of the barcodes are not uniform, and the packaging materials to which the barcodes are attached are not all the same, which leads to the lack of a good recognition rate for the class of nerves, and identification is required. Time is not as fast as expected.

Previously, a laser scanner is used to read barcodes. One of the primary problems is the scanner response range. As a matter of fact, we try to propose a method to the problem of barcode recognition using the machine vision technique. In this paper we focus on the recognition of Code 39. It is due to that the Code 39 has some kinds of symbols to be utilized and it will be very practical to use the machine vision technique.

This paper introduces a bar code automatic positioning system that uses a series of image pre-processing, morphological calculations, and uses the linear characteristics of bar code images. It is hoped that the bar code position information can be obtained directly from the image to facilitate further application in identification work.

\section{BARCODE RECOGNITION}

Generally, the images of barcodes are obtained from CCD and converted into grey-scale image. The most important step of this successive process is selecting a suitable judge value for threshold. If we select a bad value, the characters shall be losing. Otherwise, select a perfect judge value, the threshold image is not only bring out the barcode characters but also assist the recognition to identification a useful and satisfied consequence. The identification flow chart is shown as in Figure 1.

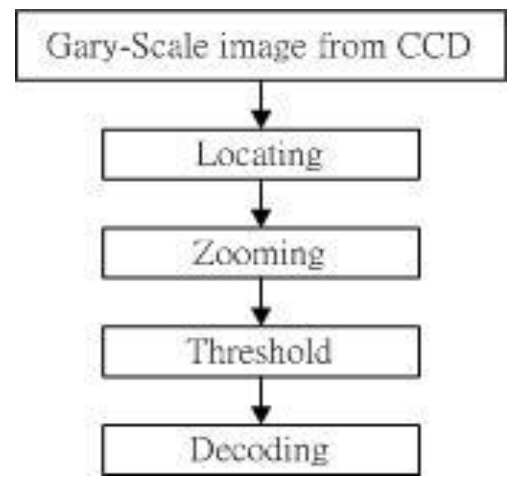

Fig. 1. The flow chart for barcode recognition.

\section{A. Image Processing}

To find the bar code image from the image, you must first perform a series of enhancement and highlight processing on the image. The brightness data of the color image is extracted through calculation, so that the entire image is converted from the original color to a single-tone grayscale image, thereby reducing the amount of image data that needs to be processed and speeding up subsequent processing.

Since the obtained image comes from the general environment, it will be interfered by some uncontrollable factors such as light, heat and electromagnetic. These interferences will disturb the image to a certain extent, causing the output result to be wrong, and then affecting the judgment result. For this problem, digital images often use averaging filtering and median filtering to suppress noise. The median filter has a better noise suppression effect and can maintain a better profile. However, due to the sorting process in its algorithm, it is quite time-consuming. The averaging filter also has a good noise suppression effect, but its ability to save contours is poor, but because the barcode image is quite obvious and regular, the averaging filter can be used for processing, which can save a lot of computing time.

All image pixel values are classified according to a specific threshold. Those exceeding this threshold are classified as white, and those less than or equal to the threshold are classified as black. That is to say, all pixels are divided into two types: the main body and the background, so as to quickly separate the barcode main body. The threshold selection in the binarization process is quite critical, so how to make the system automatically select an appropriate threshold value needs to be explored.

There are many ways to take the binarization threshold. For example, the Otsu [7] method is the most commonly used method, which performs binarization threshold calculation on each horizontal line in the cut image. When processing grayscale images, due to the grayscale value distribution of the bar code itself, there will be a big height difference like a canyon. Therefore, using this characteristic application, this paper proposes linear binarization, but its disadvantage is that it will be time-consuming in operation. For example, on a $640 \times 480$ pixel image, after the image is gray-scaled, this method will generate 480 thresholds to provide binarization for each horizontal line on the image. Since there may be noise around the barcode during identification, this binary method can highlight the features on the horizontal line of the barcode. As shown in Figure 3, the areas on the left and right sides of the barcode are clean and free of noise. Steps as following:

Step1: When we scan the image, we calculate every transverse line in the same image. Assume that image size is $800 \times 600$ pixels, using the following equation (1), that image will calculate 600 times and arise six hundreds of judge value for every transverse line. In equation (1), " $y(i)$ " is the judge value for every transverse line, " $w$ " is target image width. And " $f(x, y)$ " is one pixel in target image. This formula plus all grey level pixels in one transverse 
line. And then divide the value by image width and you'll obtain the judge value.

$$
y(i)=\frac{\sum_{x=1}^{w} f(x, y)}{w} \quad x, i \in N
$$

Step2: After selecting the judge value by equation (1), processing whole image. Figure 2 shows the result.

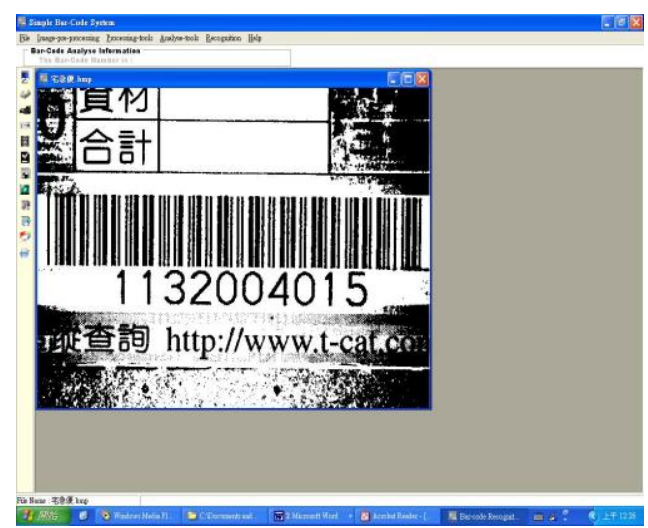

Fig. 2. Threshold at average judge value.

Because of the barcode features are linear characters, this arithmetic will very useful. It makes the feature easier to give a description of the barcode.

\section{B. Morphological Opaerations}

The image pre-processing used in this article is mainly to binarize the obtained grayscale image to obtain black and white image data. Since the acquisition process may be affected by noise, these noises need to be removed. Here, we use typology to remove noise. Generally speaking, morphological calculations are based on the 8 neighborhood pixels around the pixel as the basis for processing judgment. However, due to the linear characteristics of the barcode, we only take the upper and lower neighborhood pixels as the intermediate pixels. The basis for judgment. This not only shortens the morphological calculation time, but also highlights the greatest feature of barcode linearity. This method is not only feasible and fast for simpler images. In the calculation of morphology, the method of first dilation and then erosion is adopted. After repeated erosion and expansion operations, only the barcode part and some small noises are left in the image.

Bar code has a special characteristic of linear morphology. Amould et al. [1] used the special black-and-white image characteristics of the bar code, and the grayscale value changes very drastically. Therefore, special processing was performed on the image to eliminate unnecessary noise. Because of this hint, a linear morphological operation is proposed for barcode image positioning. The morphological calculation method is to take the 8 pixels around the pixel as its judgment basis. However, due to the linear characteristics of the barcode, after the obtained grayscale image is binarized, the surrounding 8 pixels are not selected, only the above And the two pixels below are used as the basis for judging the intermediate point, which not only shortens the morphological calculation time, but also highlights the biggest feature of the barcode. This method is not only feasible and fast for simpler images.
By combining the Dilation and Erosion operations in morphology, the opening operation (erodes first and then dilation) is formed. Through the opening operation, the contours in the image can be smoothed and the dot-like impurities can be eliminated. Information, to facilitate subsequent related processing, and then the details are described as follows.

Dilation is one of the most basic operands in mathematical typology. In the plane, there are two images A and B. The definition of Dilation of $\mathrm{A}$ and $\mathrm{B}$ is shown in equation (2).

$$
D(A, B)=A \oplus B=\bigcup_{b \in B} A+B
$$

$\mathrm{A} \oplus \mathrm{B}$ can be regarded as the union of the result obtained by translation of each element in B by A, and B is the structural element. Figure 3 shows the result of Dilation between a rectangle $\mathrm{A}$ and a structural element $\mathrm{B}$. If $\mathrm{B}$ is symmetrical about the origin, an expansion effect can be produced.
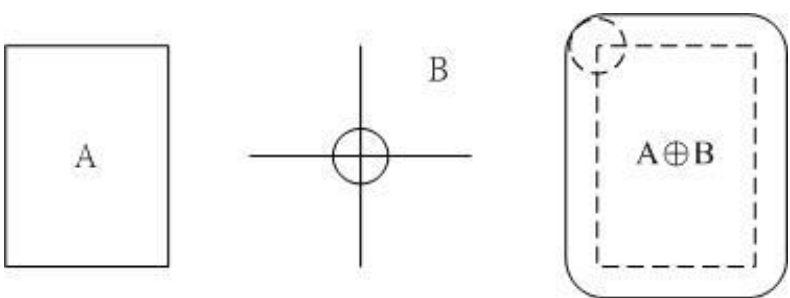

Fig. 3. Dilation between A and B will have the effect of expanding.

Another basic operand in morphology is erosion [4]. In the plane, there are two images $\mathrm{A}$ and $\mathrm{B}$. Then the definition of erosion of $\mathrm{A}$ and $\mathrm{B}$ is shown in equation (3).

$$
E(A, B)=A \Theta B=\bigcap_{b \in B} A-B
$$

Figure 4 shows the result of Erosion for A and B. A is a rectangle, and the structural element is a line segment. The result of erosion is the oblique area in the figure. When $\mathrm{B}$ takes the origin as the symmetrical point, a shrinking effect will occur.
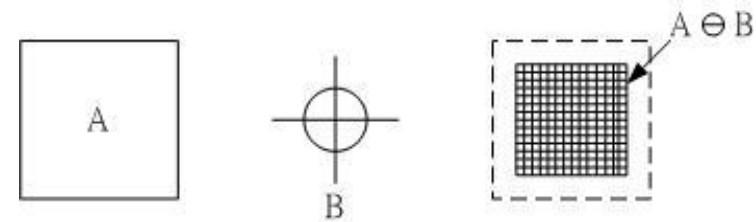

Fig. 4. Erosion of A and B will produce shrinkage erosion effect.

By combining the expansion and erosion in the digital image, the opening operation is formed. By turning on the calculation, the contours of the objects in the image can be smoothed. Through this feature, the surface contour of the object can be smoothed, which is convenient for subsequent related processing.

\section{Projections}

Repeated projection is a recursive process. First, the image is roughly divided in a simple projection method, and then the number of objects is detected for each divided block. If the number of objects in the divided area exceeds two or more, then perform simple projection on this area, and then detect the number of objects in each area obtained from the second division. If there are more than two areas (including two), 
continue to divide by simple projection Otherwise, stop the projection division operation for this area. This process is repeated iteratively until there is no area that can separate more than two objects. The advantage of this method is that it can divide objects of different sizes and scattered in disorder, and this feature is just suitable for general barcode images.

After 15 continuous morphological calculations, and finally an open calculation, the original image is only left with a barcode and some small noises. At this time, the projection method can be used to accumulate black pixels in the horizontal and vertical directions. After calculating the average value, select the approximate location of the barcode.

After the barcode image is strengthened, the horizontal and vertical projection methods are then used to accumulate the pixel points of the object on the $\mathrm{X}$ and $\mathrm{Y}$ axes, and two histograms can be obtained. Then, by analyzing the two histograms, the coordinates of the area where the object is located can be obtained and the relevant features can be retrieved. Accumulate the black pixels in the horizontal and vertical directions respectively, and after calculating the average value, box select the approximate position of the barcode.

\section{Code 39 Synopsis}

There are forty-four symbols in code 39 [5]. Each symbol contains nine lines. It was used by some research because the Code 39 was very easy to encode. The black line was named "bar", and the white line was named "space".

Code 39 characters were constructed by four elements, including wide or no-wide and slender lines contain "bar" and "space". The composition of each symbol is five "bar" and four "space". In one symbol, it has nine lines. Those nine lines must consist of three wide lines and six slender lines. All of lines can be "bar" or "space".

On the other hand, there is one slender space makes a distinction between two symbols. The first and last symbol in a set of barcode is "*", such as the red rings in Figure 5. Therefore, just because the three wide lines and nine lines formation, it's named "Code 39".

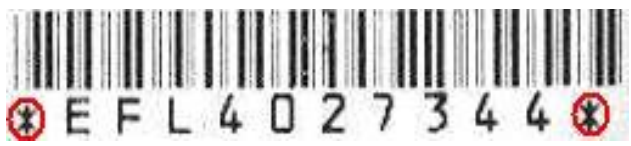

Fig. 5: A set of "Code 39".

\section{E. Define the Best Sample Line}

Assume we use whole image, the speed will be very slowly. If there is only select one line, we find the result is not very effective. Therefore, in order to accelerate the speed of recognition, we only decide using nine lines to resolve the identification when we processing the fixed position image. Finally, we average nine lines and then we will obtain one line provide decoding.

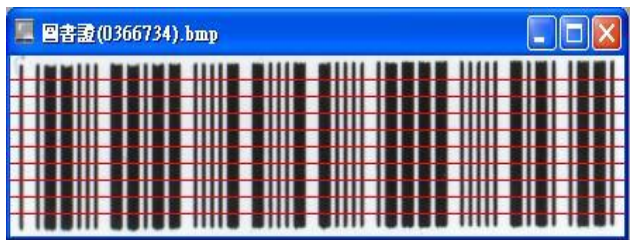

Fig. 6: The nine sample lines.
Besides, the nine lines are using median transverse line to select. And then utilize the image height divided by 10 , obtained a value. We use the value to plus and minus with half height multiply, and then gained the nine lines. The red lines in Figure 6 show the measure lines.

Assuming that during identification, the difference between scanning the entire image and scanning 9 horizontal lines must be 9 lines fast, and we call those 9 lines the sample definition line, which is used to determine the final definition line for identification. In the barcode area image, the image width is cut into 10 equal parts for the entire image. Among these 10 equal parts, 9 lines will be generated. Then the number of black pixels will be calculated to eliminate the large gap. Of the line. The so-called line segment with too large gap is defined as the occurrence of damage to the barcode image. After elimination, the number of defined lines must be less than 9, and then the remaining lines are averaged to determine the data to be used for the final identification.

\section{F. Decoding}

In recognition step, after we obtained the best sample line to analyse, we need standardize four make-up elements. There is wide "space", wide "bar", slender "space" and slender "bar" need to process. Because a set of barcode first and last symbols are "**. We use the first symbol to select the four elements. Figure 7 shows the symbol of "*". Besides, assume the wide lines is 1 , slender lines is 0 . The “*” will be"010010100".

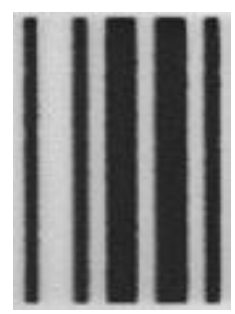

Fig. 7: The symbol of “*”,

After magnifying the position of the bar code, scan the bar code image from left to right according to the basic definition line to get the black and white and thickness data. This data can be compared with the pre-stored 39 code code principle to obtain each code. In addition, since the initial and ending symbols of each group of barcodes are "*", the two initial and ending symbols can be omitted in the decoding process.

The symbol of "*” contain one wide "space", two wide "bar", three slender "space" and three slender "bar". Therefore, we use two kinds of slender line to select four elements. We are processing as follow:

Step1: Average the three slender "space" to standardize the slender "space".

Step2: Using the standardized slender "space", multiply 1.5 to standardize the wide "space". But if the line less than the 0.8 times of slender "space", we will omitted it.

Step3: Average the three slender "bar" to standardize the slender "bar".

Step4: Using the standardized slender "bar", multiply 1.7 to standardize the wide "bar". Because of most slender "bar" of product are very thin. Therefore, we select a 
higher multiple to calculate. But if the line less than the 0.8 times of slender "bar", we'll omitted it.

After standardize all width, we can use nine widths to identification every symbol.

\section{G. Zooming and Flipping}

Assume the barcode image from CCD is too small; we may lose some important features and this lose brought about the result fail. As a result, we try to magnify the image at an opportune moment. Both the width and height are two times. It can make features clearer. Besides, in some situation, the image reverse possibility, flipping can correct the mistake.

In the analysis process, the image after positioning and cutting is sometimes too small. Therefore, in order to improve the accuracy, this article enlarges the image graphics. Although it will increase the analysis time, the processing of the image after cutting is already defined. It is processed when the line is in use, and only the 9 lines to be used are processed, so the speed will increase a lot. The biggest advantage of magnification is that it magnifies the features of the bar code, which can increase the recognition rate. This article uses $2 x$ magnification.

All image pixel values are classified according to a specific threshold. Those exceeding this threshold are classified as white, and those less than or equal to the threshold are classified as black. That is to say, all pixels are divided into two types: the main body and the background, so as to quickly separate the barcode main body. The threshold selection in the binarization process is quite critical, so how to make the system automatically select an appropriate threshold value needs to be explored.

\section{H. Barcode Database}

There are one or more data tables in a database, and there are one or more data columns in the data table, and the actual data is stored in the data column. A management system must pay attention to several things: the interface is appropriate, the effect is remarkable, the real-time service, and the stability and compatibility. If it can reduce the cost and reduce the construction cost, it will increase the user's chance of adoption. Both Access and Excel in Microsoft office are currently widely used software. The latter has the functions of easy modification and mathematical function calculations, while the former has more professional functions, which can quickly search out what you want from a large amount of data. For data and related information, the former is a good choice for users who have a strong need for quick search and do not need the trial calculation function.

ODBC (Open Database Connectivity) is a database standard interface defined by Microsoft. As long as the database software used by the user provides an ODBC driver, we can allow applications to access these supported ODBC databases. Because the file format used by each database software is not the same, our applications or systems developed by our own programs, if you want to access these databases, of course, you need a driver in the middle. The medium, as if there is a mouse driver to control the mouse, use ODBC to achieve the purpose of accessing the database.

\section{EXPERIMETNAL RESULTS}

To evaluate the proposed method, one thousand of barcodes have been tested. The experiment results show that the decoding method may be varied. Further, some features may be lost in the feature extraction step. To solve the problem, we can magnify the image before identification.

Just because lose some feature, it makes the recognition can't work. As a result, we try to magnify the image at an opportune moment. The table 1 shows the result is very good after magnified. The identification ratio improves so much and it promotes $25 \%$. Therefore, the result proves our method is a feasible scheme. Figure 8 shows the recognition result of barcode.

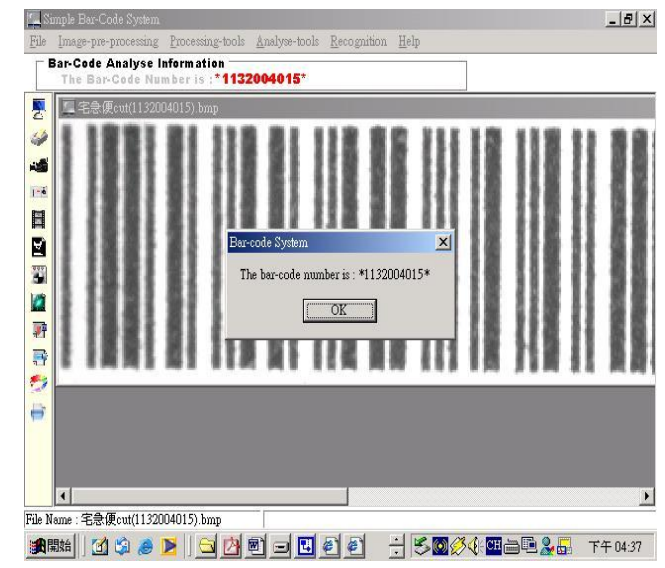

Fig. 8. The recognition result of barcode.

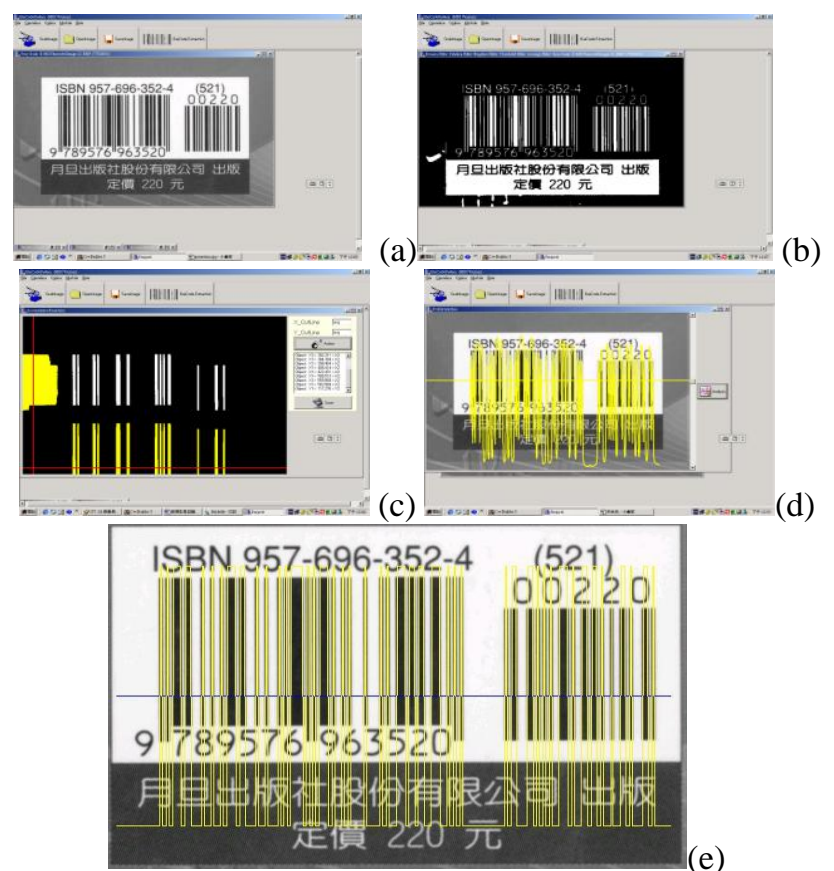

Fig. 9. (a) After the original barcode image is processed with gray scale, (b) the image after averaging filtering and opening operation, (c) after the area ratio filtering method, then the histogram image obtained by cumulative projection, (d) perform cross-hatching analysis on the detected barcode area, (e) the barcode characteristic analysis line after the cross-hatching is polarized

The method proposed in this research obtains the barcode width polarized by the cross-section line, and divides all other barcode objects in the same image with the largest width in 
the barcode group obtained in each image Width, the barcode width data is normalized in this way. From the experimental results, it is not difficult to find that once the background of the barcode image becomes complicated, the image after the binarization will easily leave many residual images. Although the filtering process mentioned in this article can eliminate most of the residual images, some solid residual image blocks will still remain, which will interfere with the accuracy of barcode analysis. Therefore, how to find an effective binarization method is one of the directions that can be discussed in depth. On the other hand, the current detection of bar code images is based on a specific direction of detection. For the detection of different angles, it still needs to be defined in advance by personnel, or multi-directional detection, so how to detect freely rotating bar code images is strengthened. The adaptability and flexibility of the system is another direction that can be explored. Figure 9 is a continuous schematic diagram of the entire barcode feature analysis system.

\section{CONCLUSIONS}

This paper proposes a simple barcode feature analysis system, which reduces the amount of processed data by graying the barcode image, then suppresses noise with average filtering, and then binarizes the image with the thresholding method, and then uses the closed operation to smooth the outline of the barcode body. Then select the object in the image by repeated projection, by judging whether the proportion of the object in the box has reached the threshold, if there is, the object in the box is retained, otherwise it is eliminated, and filtered by this method Unless the barcode image. Subsequently, the filtered images are accumulated and analyzed by cumulative projection, and then the coordinates of the area where the barcode is located are obtained. Finally, by analyzing the profile of the area where the barcode is located, and polarizing the waveform obtained, the width information of the barcode is read, and after normalization, with the calculation of the encoding rules, the content of the barcode in the image can be calculated. This method is fast and practical.

\section{REFERENCES}

[1] S. Arnould, G. J. Awcock, and R. Thomas, "Remote bar-code localization using mathematical morphology", Proceedings of IEE Seventh International Conference on Image Processing and Its Applications, 2, July, 1999, pp. 642-646.

[2] R. J. Howlett, S. Berthier, and G. J. Awcock, Determining the location of industrial bar codes using neural networks, Proceedings of the Sixth International Conference on Image Processing and Its Applications, 1997, Vol. 2, pp.511 -515.

[3] A. K. Jain and Y. Chen, "Bar code localization using texture analysis", Proceedings of the Second IEEE International Conference on Document Analysis and Recognition, 1993, pp. 41-44.

[4] S.J. Liu, H.Y. Liao, L.H. Chen, H.R. Tyan, and J.W. Hsieh, "Camera-based bar code recognition system using neural net", Proceedings of 1993 IEEE International Joint Conference on Neural Networks, 2, 1993, pp. 1301-1305.

[5] R. Muniz, L. Junco, and A. Otero, "A robust software barcode reader using the Hough transform, "IEEE Bethesda, MD, USA, 1999, pp. 313-319.

[6] E. Ohbuchi, H. Hanaizumi, L. A. Hock, L.A., Barcode readers using the camera device in mobile phones, 2004 IEEE International Conference on Cyberworlds, 18-20 Nov. 2004, pp. 260-265.

[7] N. Otsu, "A threshold selection method from gray-scale histograms, “IEEE Trans. System, Man, and Cybernetics 9(1), 1979, pp. 62-66.
[8] C. Viard-Gaudin, N. Nicolas, and D. Barba, A bar code location algorithm using a two-dimensional approach, Proceedings of the Second International Conference on Document Analysis and Recognition, 1993, pp.45 -48.R. Muniz, L. Junco, and A. Otero, “A robust software barcode reader using the Hough transform, " IEEE Bethesda, MD, USA, 1999, pp. 313-319.

Wen-Yen Wu is a Professor of Industrial Management at I-Shou University, Kaohsiung, Taiwan, ROC, since 2001. His research interests include automated inspection, machine vision, fuzzy set theory, pattern recognition, and anthropometric applications. Dr. Wu is a member of the Phi Tau Phi, the Chinese IPPR Society, the Chinese Institute of Industrial Engineers, the Ergonomics Society of Taiwan, the Chinese Fuzzy Systems Association, the Distribution Management Society, Taiwan, and the Chinese Institute of Automation Engineers. 